\title{
Escoliose Distrófica na \\ Neurofibromatose Tipo I
}

Dystrophic Scoliosis in Neurofibromatosis Type I

Germano Nascimento, Jorge Mineiro, João Cannas, Luís Barroso

Autor Correspondente:

\section{RESUMO}

A neurofibromatose tipo 1 (NF-I) é uma patologia hereditária autossómica dominante, na qual deformidades da coluna vertebral podem manifestar-se em idades muito precoces. A escoliose distrófica manifesta-se habitualmente como deformidade adquirida muito acentuada, com evolução progressiva e frequentemente com necessidade de correção cirúrgica. Os autores reportam o caso raro de um doente de 14 anos com escoliose distrófica progressiva, tratado nesta instituição. O resultado obtido foi muito satisfatório e pretende-se acrescentar orientação clínica à literatura científica atual.

PALAVRAS-CHAVE: Adolescente; Escoliose/cirurgia; Neurofibromatose 1/cirurgia

\section{ABSTRACT}

Neurofibromatosis type I (NF-I) is a dominant autosomal hereditary pathology, where deformations of the back bone appear in very young ages. Dystrophic scoliosis frequently appears as a very pronounced deformity, which gets progressively worse and frequently needs surgical correction. The authors report the rare case of a 14-year-old patient with progressive dystrophic scoliosis, who was treated in this institution. The result was very satisfactory and the authors intend to add clinical guidance to the current scientific literature.

KEYWORDS: Adolescent; Neurofibromatosis 1/surgery; Scoliosis/surgery 


\section{INTRODUÇÃO}

A neurofibromatose (NF) representa uma das patologias genéticas mais comuns, afetando cerca de 1 em cada 3.000 pessoas. ${ }^{1}$ Tratando-se de uma doença autossómica dominante, tem uma expressividade variável com 100\% de penetrância e alto índice de mutação. Atualmente são aceites pelos investigadores e descritas na literatura 3 formas desta doença: NF tipo 1 (periférica, com lesões cutâneas típicas em café-au-lait e associada a manifestações ortopédicas), NF tipo 2 (central, associada a schwannomas vestibular e espinhal) e uma terceira forma, NF segmentar, mosaico da NF tipo $1{ }^{2}$

Na neurofibromatose tipo 1 as manifestações ortopédicas são frequentes, sendo a escoliose evidente em até cerca de 60\% dos doentes afetados. ${ }^{3}$ Esta pode manifestar-se de duas formas: deformidades distróficas e deformidades não distróficas.

As deformidades não distróficas simulam a escoliose idiopática, habitualmente com curvaturas pouco acentuadas, sendo reservado o tratamento cirúrgico para os casos mais graves e com curvaturas superiores a $40^{\circ}$.

As deformidades distróficas são mais frequentes e caracterizam esta doença. São identificadas radiograficamente quando observada a deformidade vertebral associada a curto raio com grande aumento de rotação, defeito nos pedículos vertebrais, luxação costovertebral associada a costela afilada em "ponta de lápis". A deformidade pode ser agrupada de acordo com o seu perfil sagital, podendo ser normal, cifótica ou lordótica. ${ }^{4}$

A progressão clínica é tanto mais célere quanto mais grave for a alteração distrófica verificada. Apesar de ser aceite durante muito tempo, o tratamento conservador foi demonstrado ser ineficaz nestes pacientes, sendo o tratamento cirúrgico atualmente a opção de eleição. Apesar do planeamento cuidado e minucioso, existem várias complicações descritas para este procedimento, mesmo em doentes sem défice neurológico prévio. 5

Para abordar este tema propomos a apresentação de um caso clínico tratado nesta instituição, elucidando o percurso diagnóstico e terapêutico efetuado.

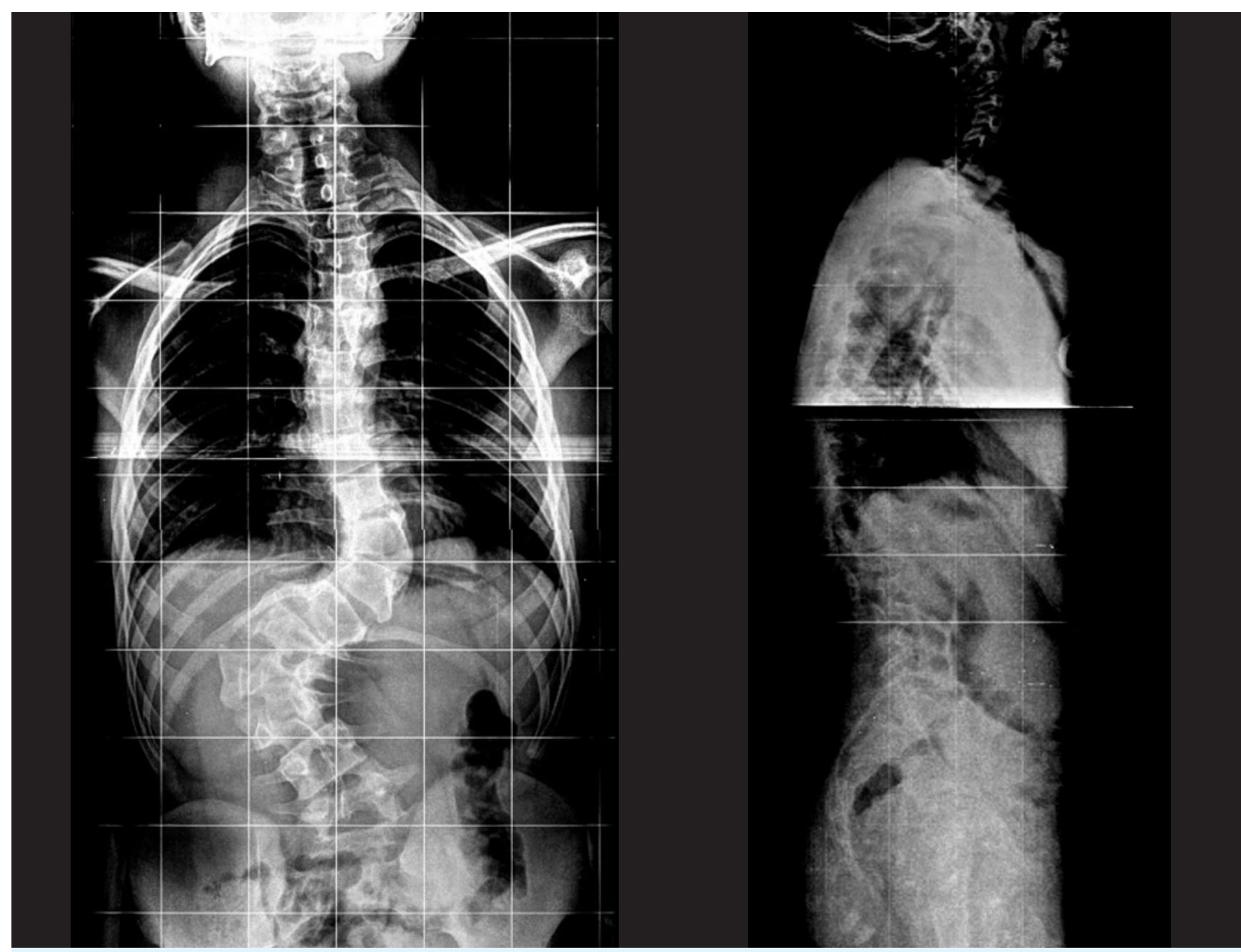

FIGURA 1. Telerradiografia da coluna vertebral em posição ântero-posterior e de perfil, aquando da primeira observação do doente. É notória a acentuação da curvatura no plano sagital. 


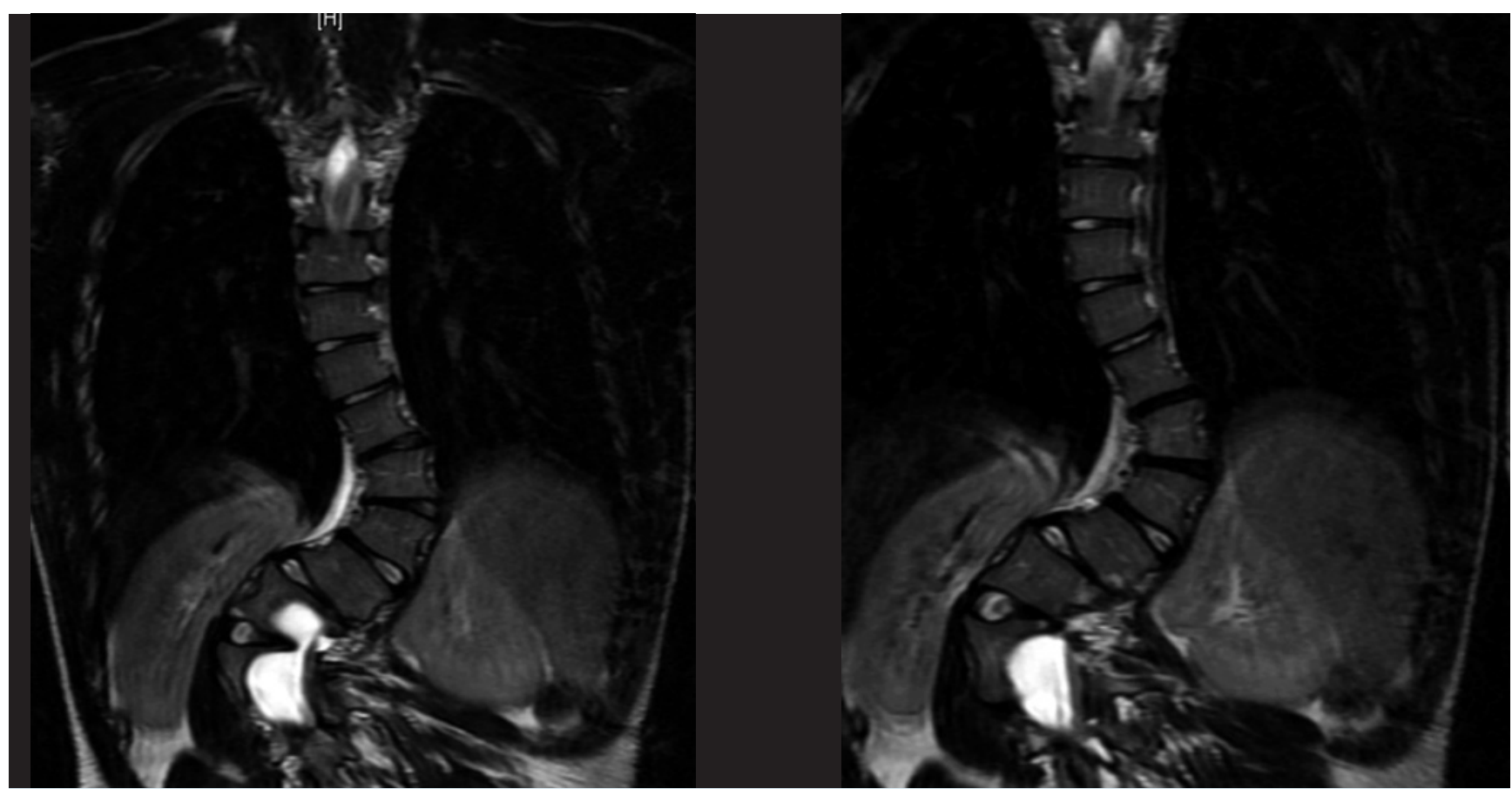

FIGURA 2. Imagens obtidas por ressonância magnética nuclear. Não foram identificadas lesões neurológicas associadas à deformidade da coluna tóraco-lombar.

\section{CASO CLÍNICO}

PANF, doente do sexo masculino, 14 anos de idade, foi referenciado à nossa instituição por deformidade dorsal progressiva. Tratava-se de um doente com antecedentes conhecidos de Neurofibromatose tipo 1. Aquando do exame clínico inicial, o doente apresentava-se consciente, colaborante e orientado.

Apesar da deformidade, o doente negava inicialmente qualquer tipo de dor, cefaleias suboccipitais, parestesias, retenção urinária ou outras alterações do foro neurológico.

Objetivamente, além das múltiplas lesões cutâneas típicas em café-au-lait, era visível a presença de uma tumefação torácica posterior, hipersensível, correspondente a um neurofibroma.

A deformidade determinava uma gibosidade lombar alta, com alguma mobilidade na hiperextensão do tronco. A curvatura estava clinicamente compensada.

A força muscular da anca e joelhos era normal, e não se verificaram alterações da sensibilidade dos membros inferiores, com reflexos normais e sinal de Babinski negativo.

O doente não tinha outras alterações, nomeadamente cerebrais, oftalmológicas e cardiológicas.

Inicialmente observado a 5-12-2012 » indicação cirúrgica a 12-6-2013 » operado a 24-8-2013 » última observação a 14-10-2014.
O estudo radiológico (Fig. 1) demonstrou a presença de escoliose dismórfica com curvatura de $96^{\circ}$. Foi realizado consecutivamente um estudo por ressonância magnética (Fig. 2), que não revelou qualquer tipo de lesão da medula espinhal e das suas raízes. $\bigcirc$ estudo por tomografia computorizada permitiu o planeamento cirúrgico e a determinação da configuração óssea necessária para uma correção nos planos coronais e sagitais.

O doente foi operado a 24 de agosto de 2013, tendo sido realizada fusão intersomática após discectomia de T12, L1 e L2 por via tóraco-freno-laparotomia por via extra-pleural para libertação da curvatura e excisão dos discos intervertebrais no apex da curvatura e para otimizar a fusão intersomática, tendo em conta que este tipo de doentes têm uma taxa mais elevada de falência da artrodese posterior. De seguida, sob a mesma anestesia geral reposicionou-se o doente e procedeu-se à instrumentação de T4 a L4 com parafusos pediculares por via posterior com facectomias a todos os níveis e colocação de enxerto autólogo para correção da deformidade.

A cirurgia foi realizada sob monitorização intraoperatória de potenciais evocados motores e somatossensitivos (Fig. 3).

O pós-operatório decorreu sem intercorrências, tendo o doente tido alta ao $7^{\circ}$ dia após a intervenção. Foi sujeito a um programa orientado de reabilitação com Fisioterapia precoce, determinando uma progressiva meIhoria clínica verificada durante o seguimento. $\bigcirc$ estudo radiológico (Fig. 4) revelou uma correção satisfatória e estabilização da coluna vertebral. 

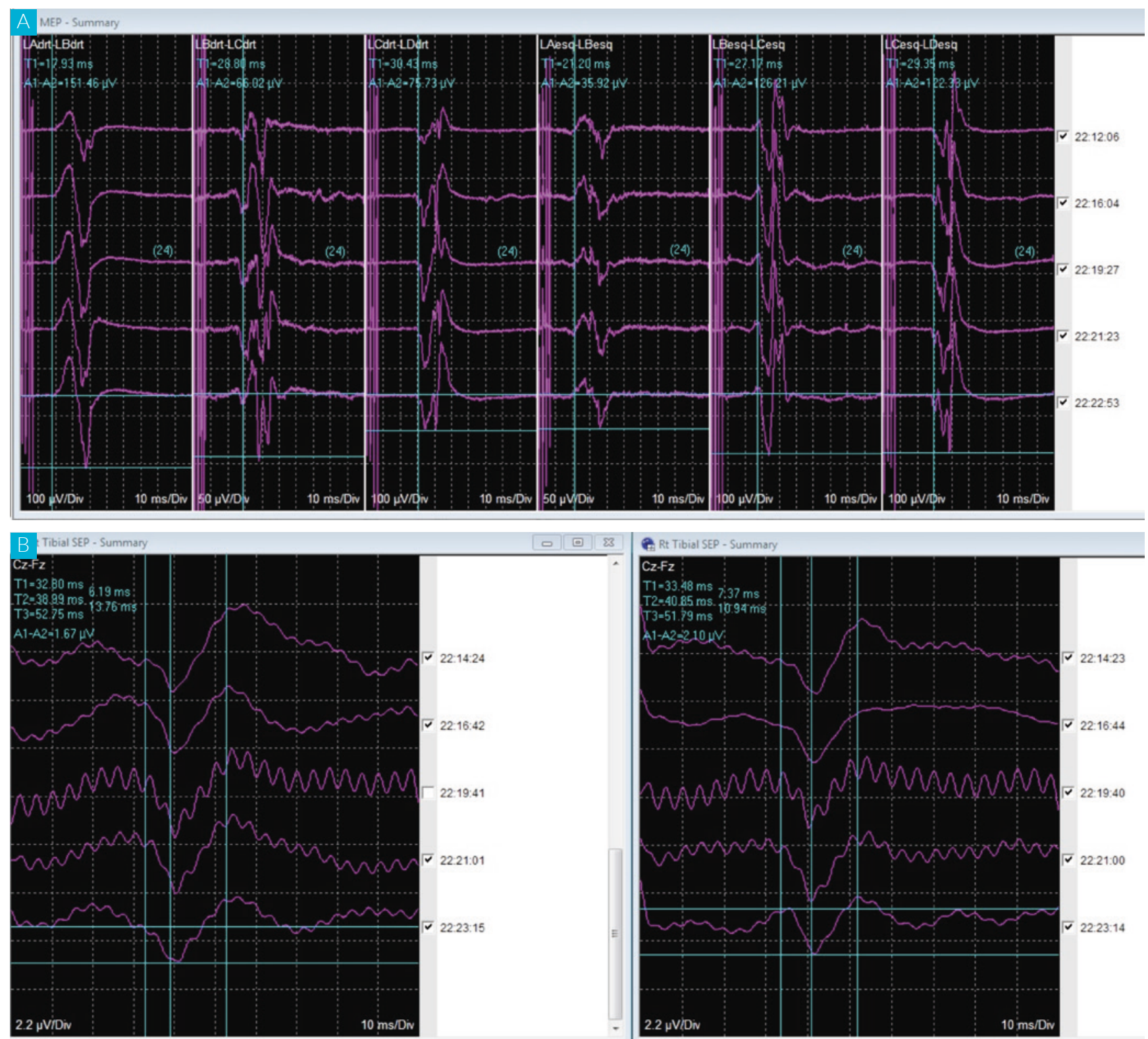

FIGURA 3. Registo da monitorização neurofisiológica realizada intraoperatoriamente. Trata-se de parte das aquisições realizadas, sendo representativas da totalidade das mesmas: potenciais evocados motores (A) e somatossensitivos (B).

Dezasseis meses após a intervenção, o doente estava clinicamente sem dores e não tinha limitações nas suas atividades diárias.

\section{DISCUSSÃO}

As afeções ortopédicas encontradas na neurofibromatose tipo 1 compreendem frequentemente a presença de escoliose do tipo distrófica, com manifestação precoce na vida do doente com esta patologia.

O tratamento cirúrgico é fundamental na preservação de uma normal fisionomia, evitando as complicações tidas como certas no desenvolvimento e progressão na história natural da doença.

Tratando-se de um procedimento minucioso e tecnicamente difícil, deve ser realizado por profissionais com elevada experiência na correção de deformidades da coluna vertebral, em centros cirúrgicos de excelência aliados a equipas multidisciplinares dedicadas. ${ }^{6}$

Uma das dificuldades associadas a esta intervenção, tanto mais prevalente quanto maior a progressão da doença, consiste na instrumentação segmentar com vista à fusão, devido ao pobre stock ósseo em virtude da destruição vertebral subsequente.? Esta é a razão pela qual neste tipo de patologia é sempre mais seguro proceder-se também a artrodese intersomática por via anterior no mesmo tempo operatório ou em diferido, dependendo de cada caso.

O caso aqui descrito demonstra um resultado muito positivo perante uma situação clínica complexa e de tratamento cirúrgico exigente. 


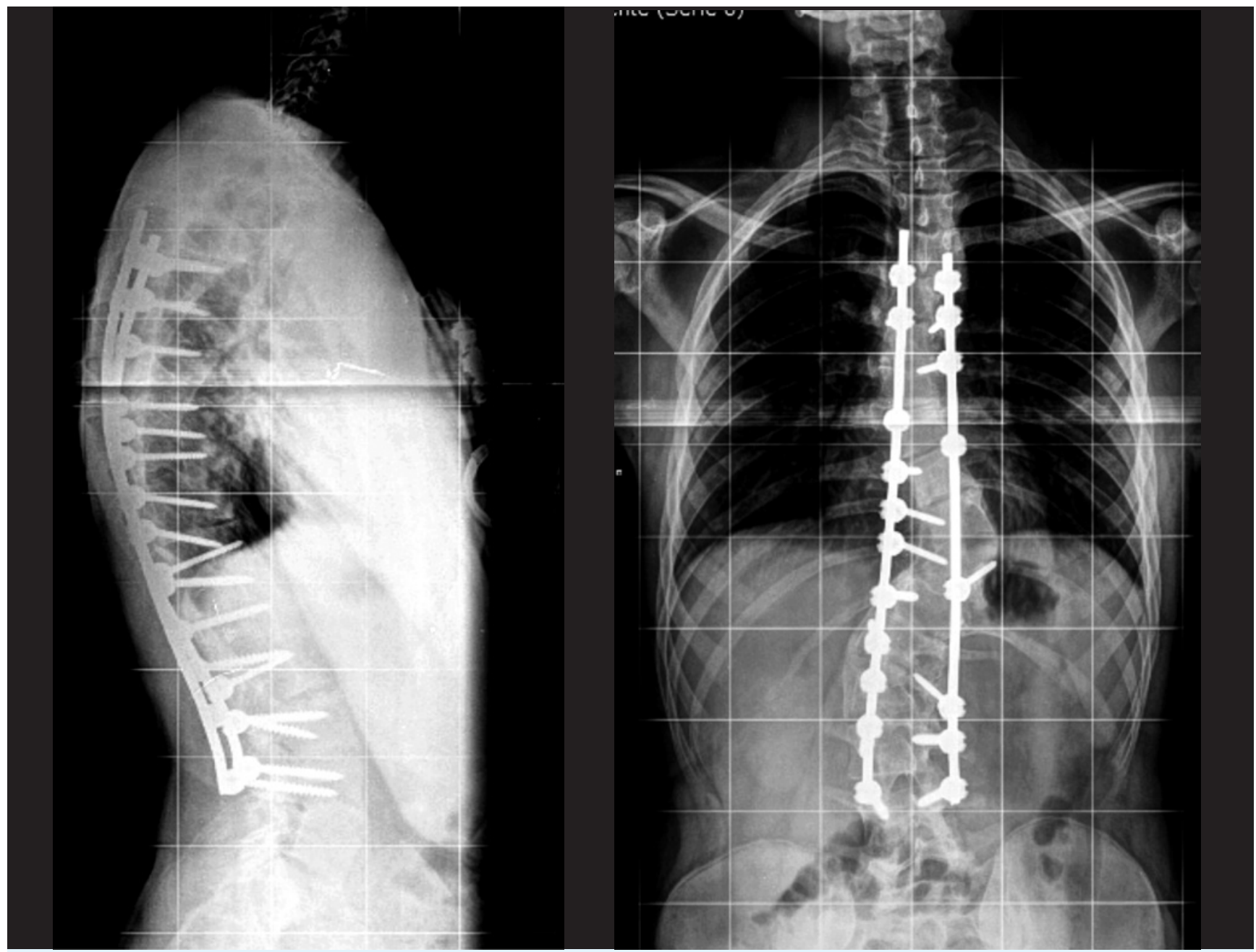

FIGURA 4. Imagens radiográficas obtidas na última consulta de seguimento. A correção da deformidade obtida é muito satisfatória. É notória uma estabilização da coluna vertebral, sem falência ou rejeição dos implantes.

Devido à raridade desta patologia, a literatura é deficitária no que concerne à abordagem gold standard relativamente a esta situação clínica. Este caso tem a intenção de acrescentar evidência científica quanto ao tratamento da escoliose distrófica nos doentes com neurofibromatose tipo 1.

CONFLITOS DE INTERESSE: Os autores declaram a inexistência de conflitos de interesse na realização do trabalho.

FONTES DE FINANCIAMENTO: Não existiram fontes externas de financiamento para a realização deste artigo.

PROTEÇÃO DE PESSOAS E ANIMAIS: Os autores declaram que os procedimentos seguidos estavam de acordo com os regulamentos estabelecidos pelos responsáveis da Comissão de Investigação Clínica e Ética e de acordo com a Declaração de Helsínquia da Associação Médica Mundial.

CONFIDENCIALIDADE DOS DADOS: Os autores declaram ter seguido os protocolos do seu centro de trabaIho acerca da publicação dos dados de doentes.

\section{REFERÊNCIAS}

1. Crawford AH, Parikh S, Schorry EK, Von Stein D. The immature spine in type-1 neurofibromatosis. J Bone Joint Surg Am. 2007; 89 Suppl 1:123-42.

2. Gerber PA, Antal AS, Neumann NJ, Homey B, Matuschek C, Peiper M, Budach W, Bölke E. Neurofibromatosis. Eur J Med Res. 2009;14:102-5.

3. Koptan W, EIMiligui Y. Surgical correction of severe dystrophic neurofibromatosis scoliosis: an experience of 32 cases. Eur Spine J. 2010;19:1569-75.

4. Vandenbroucke J, van Ooy A, Geukers C, van der Linden AJ, Hoogmartens M. Dystrophic kyphoscoliosis in neurofibromatosis type I: a report of two cases and review of the literature. Eur Spine J. 1997;6:273-7.

5. Meneses-Quintero D, Alvarado-Gómez F, Alcalá-Cerra. G Dystrophic thoracic spine dislocation associated with type-1 neurofibromatosis: Case report and rationale for treatment. J Craniovertebr Junction Spine. 2015;6:79-82.

6. Bamps S, Van Calenbergh F, Van Loon J, Van Paesschen $\mathrm{R}$, and Vanderschot P. Posterolateral approach in a neurofibromatosis type-I patient with severe dystrophic thoracic kyphoscoliosis: A case report, cadaver study, and literature review. J Neurosci Rural Pract. 2015;6: 84-86.

7. Greggi T, Martikos K. Surgical treatment of early onset scoliosis in neurofibromatosis. Stud Health Technol Inform. 2012;176:330-3. 